\title{
ОБ ОДНОМ ЭКСПЕРИМЕНТЕ ЯМР, ВЫЯВЛЯЮЩЕМ СВЯЗЬ РЕДКИХ ЯДЕР С ИХ СОСЕДЯМИ В ТВЕРДЫХ ТЕЛАХ
}

Обмен поляризациями между многочисленными спинами $I$ и редкими спинами $S$ в твердых телах при выполнении условия ХартманнаХана $\left[{ }^{1}\right]$ носит осцилляционный характер $\left[{ }^{2,3}\right]$, обязанный членам типа $a_{n m} \mathbf{I}_{n \pm} \mathbf{S}_{m \mp}$ в гамильтониане спин-системы. Эти члены, описывающие связь между $I$ - и $S$-спинами, обусловлены диполь-дипольным и спинспиновым взаимодействиями и, оџевидно, прежде всего взаимодействиями между $S$-спином и ближайшими к нему $I$-спинами. Появление осцилляций позволяет определить величину связи и, тем самым, получить ценную информацию о ближайшем окружении редких спинов в твердых телах $\left[{ }^{2,3}\right]$. Однако реализация подобного эксперимента связана с рядом технических и интерпретационных трудностей [ [ $\left.{ }^{4}\right]$.

В настоящей статье теоретически рассматривается один из вариантов эксперимента ЯМР, позволяющий также определить связь редкого спина с его соседями. Основная идея его (высказанная Дж. Уо $\left[{ }^{5}\right]$ ) заключается в следующем: за определенный промежуток времени неравновесная намагниченность $S$-спина изменяется в условиях спинлокинга $I$-спинов под магическим углом. В это время эффективно устраняется диполь-дипольное взаимодействие между I-спинами [ $\left.{ }^{6}\right]$, являющееся основным способом диффузии энергии в системе $I$-спинов. C момента окончания спин-локинга регистрируется сигнал $S$-спина в условиях развязки его от $I$-спинов $\left[^{7}\right]$. Положения линий в спектре двукратного преобразования Фурье $\left[{ }^{8}\right]$ полученного сигнала определяются константами связи $S$-спина с ближайшими соседями.

Результаты эксперимента, отличающегося от вышеописанного, в основном, только применением последовательности из 4-импульсных циклов [ $\left.{ }^{9}\right]$ вместо спин-локинга под магическим углом, приведены в $\left[{ }^{4}\right]$.

\section{1. Постановка задачи}

Рассматриваемый эксперимент состоит из следующих трех этапов:

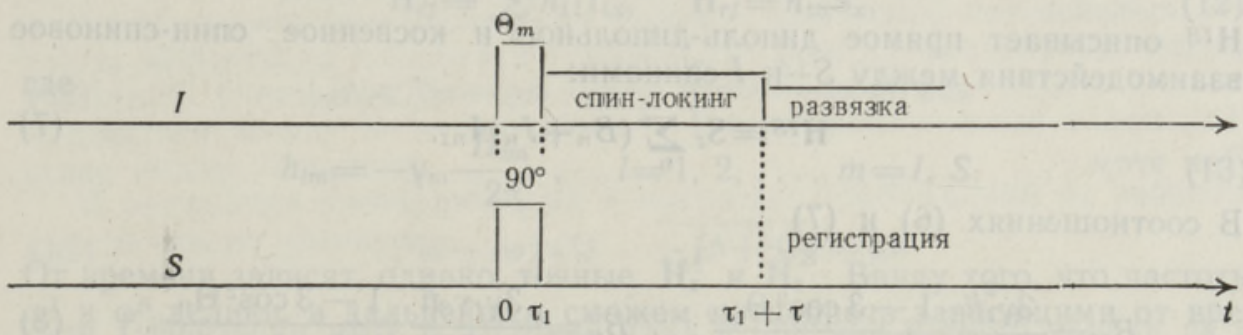

Схема эксперимента 
I. Подготовительный этап: на спин-систему, находящуюся в равновесном состоянии в сильном постоянном магнитном поле, воздействуют два ВЧ импульса, один из которых поворачивает намагниченность $S$-спинов на $90^{\circ}$, а другой изменяет намагниченность $I$-спинов на магический угол $\Theta_{m}$.

II. Спин-локинг: в течение времени $\tau I$-спины подвергаются воздействию ВЧ поля, обеспечивающего выполнение условия т. н. опинлокинга под магическим углом.

III. Р ег и с т а ц и я I-спины под воздействием сильного ВЧ поля развязываются от $S$-спинов, и сигнал последних регистрируется.

Происходящие при этом изменения оператора плотности $е$, характеризующего состояние спин-системы, подчиняется уравнению

$$
\dot{\varrho}=-\mathrm{i} \Omega(\mathbf{H}) \mathrm{\varrho} \text {. }
$$

Действие супероператора $\mathcal{Q}(\mathbf{H})$ на любой оператор $\mathbf{Q}$ соответствует определению

$$
\mathfrak{L}(\mathbf{H}) \mathbf{Q}=[\mathbf{H}, \mathbf{Q}],
$$

где $\mathbf{H}$ является гамильтонианом системы.

Во внешних сильном постоянном (z-направление) и ВЧ полях гамильтониан $\mathbf{H}$ выбирается в виде суммы нескольких слагаемых

$$
\mathbf{H}=\mathbf{H}_{z}^{I}+\mathbf{H}_{z}^{S}+\mathbf{H}_{d}^{I I}+\mathbf{H}^{I S}+\mathbf{H}_{r f}^{I}(t)+\mathbf{H}_{r f}^{S}(t) .
$$

Здесь первый член учитывает взаимодействие $I$-спинов с постоянным магнитным полем и косвенное спин-спиновое взаимодействие между I-спинами:

$$
\mathbf{H}_{z}^{I}=-\sum_{i} \omega^{I} \mathbf{I}_{i z}+\sum_{i} \omega^{I} \sum_{k=x, y, z} \sigma_{i k z}^{I} \mathbf{I}_{i k}+\sum_{i<j} J_{i j} \overrightarrow{\mathbf{I}}_{i} \overrightarrow{\mathbf{I}}_{j}
$$

Хотя в образце могут находиться $S$-спины различных по своему окружению типов, достаточно наблюдать за поведением лишь одного из них, предполағая, что взаимодействие между $S$-спинами вследствие их низкой концентрации отсутствует. Этим оправдывается выбор $\mathbf{H}_{z}^{S}$ в виде

$$
\mathbf{H}_{z}^{S}=-\omega^{S} \mathbf{S}_{z}+\omega^{S} \sum_{k=x, y, z} \sigma_{k z}^{S} \mathbf{S}_{k} .
$$

$\mathbf{H}_{d}^{I I}$ учитывает секулярную часть прямой диполь-дипольной связи между I-спинами:

$$
\mathbf{H}_{a}^{I I}=\sum_{i<j} B_{i j}\left(\mathbf{I}_{i z} \mathbf{I}_{j z}-\frac{1}{3} \overrightarrow{\mathbf{I}}_{i} \overrightarrow{\mathbf{I}}_{j}\right)
$$

$\mathbf{H}^{I S}$ описывает прямое диполь-дипольное и косвенное спин-спиновое взаимодействия между $S$ - и $I$-спинами:

$$
\mathbf{H}^{I S}=\mathbf{S}_{z} \sum_{n}\left(B_{n}+J_{n}\right) \mathbf{I}_{n z}
$$

В соотношениях (6) и (7)

$$
B_{i j}=\frac{3 \gamma_{I}^{2} \hbar}{2} \cdot \frac{1-3 \cos ^{2} \Theta_{i j}}{r_{i j}^{3}} ; \quad B_{n}=\frac{3 \gamma_{I} \gamma_{S} \hbar}{2} \cdot \frac{1-3 \cos ^{2} \theta_{n}}{r_{n}^{3}}
$$


Следует подчеркнуть, что гамильтониан H $^{I S}$ учитывает взаимодействие $S$-спина только с ближайшими к нему $I$-спинами, т. е. $n$ пробегает все значения, при которых либо $B_{n}$, либо $J_{n}$ достаточно велики.

Последние два члена в гамильтониане (3) описывают влияние ВЧ полей соответственно на $I$ - и $S$-спины,

Отметим, что уравнение (1) с гамильтонианом (3) не учитывает релаксации спин-системы, т. е. предполагает абсолютно жесткую кристаллическую решетку $\left[\begin{array}{l}10 \\ 1\end{array}\right.$.

В качестве равновесного в постоянном магнитном поле оператора плотности выбирается оператор

$$
\mathrm{Q}_{0}=(\mathrm{Sp} \mathbf{1})^{-1}\left[\mathbf{1}-\beta\left(\sum_{i} \omega^{I} \mathbf{I}_{i z}+\omega^{S} \mathrm{~S}_{z}\right)\right] .
$$

Как видно, в (9) учтены лишь доминирующие члены гамильтониана и высокотемпературное приближение $\left[{ }^{10}\right]$.

Содержание всех обозначений в (4)-(9) традиционное.

\section{2. Изменение состояния спин-системы во время подготовительного этапа}

Во время подготовительного этапа $\left(t=0-\tau_{1}\right)$ спин-система подвергается воздействию ВЧ полей

$$
\vec{H}(t)=\vec{i}\left(2 H_{1 I} \cos \omega^{I} t+2 H_{1 S} \cos \omega^{S} t\right) \text {. }
$$

Как известно, действие импульсов ВЧ поля часто сводится только к повороту намагниченностей на определенный угол во вращающейся системе координат. Несмотря на это, в настоящем параграфе показывается ход решения кинетического уравнения с целью письменной формулировки условий допустимости вышеназванного подхода и для последовательности изложения.

Итак, для облегчения определения состояния снин-системы к концу подготовительного этапа все операторы в (1) преобразуем по формуле

$$
\tilde{\mathbf{Q}}=\mathbf{R}^{-1} \mathbf{Q} \mathbf{R}^{+1}, \quad \mathbf{R}^{ \pm 1}=\exp \left[ \pm \mathrm{i} t\left(\omega^{I} \sum_{i} \mathbf{I}_{i z}+\omega^{S} \mathrm{~S}_{z}\right)\right]
$$

Лепко проверить, что члены гамильтонианов $\mathbf{H}_{d \supset 0}^{I I}$ и $\mathbf{H}^{I S}$, а также начальное состояние @o Коммутируют с (11).

Если пренебречь прямыми взаимодействиями ВЧ полей с частотами $\omega^{I}$ и $\omega^{S}$ на $S$ - и $I$-опины соответственно, а также членами с частотами $2 \omega^{I}$ и $2 \omega^{S}$, то $\tilde{\mathbf{H}}_{r f}^{I}$ и $\tilde{\mathbf{H}}_{r f}^{S}$ перестанут зависеть от времени

$$
\tilde{\mathbf{H}}_{r f}^{I}=\sum_{i} h_{1 I} \mathbf{I}_{i x}, \quad \tilde{\mathbf{H}}_{r f}^{\mathrm{S}}=h_{1 \mathrm{~S}} \mathrm{~S}_{x}
$$

где

$$
h_{l m}=-\gamma_{m} \frac{H_{l m}}{2 \pi}, \quad l=1,2, \ldots, m=I, S \text {. }
$$

От времени зависят, однако, точные $\tilde{\mathbf{H}}_{z}^{\underline{I}}$ и $\tilde{\mathbf{H}}_{z}^{\mathrm{s}}$. Ввиду тото, что частоты $\omega^{I}$ и $\omega^{S}$ велики, в дальнейшем сможем пренебречь зависящими от вре-

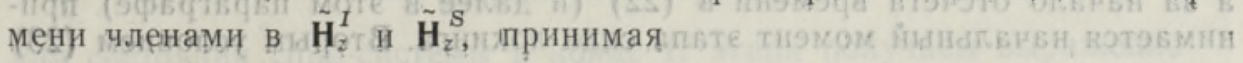




$$
\begin{aligned}
& \tilde{\mathbf{H}}_{z}^{I}=-\sum_{i} \omega^{I} \mathbf{I}_{i z}+\sum_{i} \delta_{i z z}^{I} \mathbf{I}_{i z}+\sum_{i<j} J_{i j} \overrightarrow{\mathbf{I}}_{i} \overrightarrow{\mathbf{I}}_{j}, \\
& \tilde{\mathbf{H}}_{z}^{\mathrm{S}}=-\omega^{\mathrm{S}} \mathbf{S}_{z}+\delta_{z z}^{\mathrm{S}} \mathbf{S}_{z},
\end{aligned}
$$

где

$$
\delta^{m}=\omega^{m} \sigma^{m}, \quad m=I, S .
$$

Для дальнейшего упрощения выкладок потребуем, чтобы амплитуды ВЧ полей были много больше амплитуд т. н. локальных полей, вызванных химическими сдвигами, диполь-дипольными и спин-спиновыми взаимодействиями в местах расположения любых спинов образца:

$$
\begin{aligned}
& \left|h_{1 I}\right|,\left|h_{1 \mathrm{~S}}\right| \gg\left|h_{L}\right|, \\
& h_{L}=\delta_{i z z}^{I}, \delta_{z z}^{S}, B_{i j}, B_{n}, J_{i j}, J_{n} .
\end{aligned}
$$

Подчеркнем, что условия (17) еще не оправдывают применения решения преобразованного кинетического уравнения в виде

$$
\tilde{\varrho}(t)=\mathrm{e}^{-\mathrm{i} t \Omega\left(\tilde{\mathbf{H}}_{r f}^{I}+\tilde{\mathbf{H}}_{r f}^{\mathbf{s}}\right)} \mathrm{Q}_{0},
$$

трактуемого как поворот намагниченностей во вращающейся системе координат. Чтобы (18) обрело действительный смысл, необходимо кроме того, соблюдение условия

$$
\left|h_{L} \tau_{1}\right| \ll 1 .
$$

Если, при этом, выполняются соотношения

$$
h_{1 I} \tau_{1}=\Theta_{m}, \quad h_{1} \tau_{1}=\frac{1}{2} \pi,
$$

то из (18) имеем, что в конце подготовительного этапа состояние спинсистемы выражается как

$$
\tilde{\mathrm{Q}}\left(\tau_{1}\right)=(\mathrm{Sp} \mathbf{1})^{-1}\left\{\mathbf{1}-\beta\left[\omega^{I}\left(\cos \Theta_{m} \sum_{i} \mathbf{I}_{i z}-\sin \Theta_{m} \sum_{i} \mathbf{I}_{i y}\right)-\omega^{\mathrm{S}} \mathbf{S}_{y}\right]\right\} .
$$

\section{3. Изменение состояния спин-системы во время спин-локинга}

Во время второго этапа на спин-систему воздействует ВЧ поле

$$
\overrightarrow{H(t)}=\vec{j} 2 H_{2} \cos \left(\omega_{2} t+\omega^{I} \tau_{1}\right),
$$

частота и амплитуда которого удовлетворяют условиям

$$
\cos \Theta_{m}=\frac{\Omega}{h_{e f}}=\sqrt{\frac{1}{3}} ; \quad\left|\omega_{2}-\omega^{S}\right| \gg\left|h_{2 S}\right| .
$$

При этом

$$
h_{e f}=\sqrt{\Omega^{2}+h_{2 I}^{2}} ; \quad \Omega=\omega_{2}-\omega^{I},
$$

а за начало отсчета времени в (22) (и далее в этом параграфе) принимается начальный момент этапа опин-локинга. Вторым условием (23) 
оправдывается пренебрежение прямым влиянием ВЧ поля (22) на состояние $S$-спина.

Рассмотрим изменение состояния спин-системы на II этапе. Вопервых, подвергнем все операторы $\tilde{\mathbf{Q}}$ преобразованию

$$
\mathbf{Q}_{R}=\mathbf{R}_{1}^{-1} \tilde{\mathbf{Q}}_{1}^{+1} ; \quad \mathbf{R}_{1}^{ \pm 1}=\exp \left( \pm i t \Omega \sum_{i} \mathbf{I}_{i z}\right)
$$

с целью устранить временную зависимость гамильтониана $\tilde{\mathbf{H}}_{r f}^{I}(t)$, описывающего взаимодействие ВЧ поля (22) с I-спинами. Заметим, что оператор $\tilde{\mathrm{\varrho}}\left(\tau_{1}\right)$, являющийся здесь оператором начального состояния, не изменяется с преобразованием (25). вание

Во-вторых, ко всем полученным операторам применим преобразо-

$$
\mathbf{Q}_{T}=\mathbf{T}^{-1} \mathbf{Q}_{R} \mathbf{T}^{+1} ; \quad \mathbf{T}^{ \pm 1}=\exp \left( \pm \mathrm{i} \Theta_{m} ; \sum_{i} \mathbf{I}_{i x}\right)
$$

Это преобразование можно трактовать как поворот $z$-оси по эффективному полю во вращающихся координатах. Теперь начальное состояние $\tilde{\varrho}\left(\tau_{1}\right)$ принимает вид

$$
\varrho_{T}(0)=(\operatorname{Sp~} 1)^{-1}\left[1-\beta\left(\omega^{I} \sum_{i} I_{i z}-\omega^{S} S_{y}\right)\right],
$$

а также изменяются выражения для всех членов гамильтониана.

Наконец, целесообразно провести еще одно преобразование (соответствующее вращению с частотой $h_{e f}$ вокруг $z$-оси в наклоненной системе координат)

$$
\mathbf{Q}_{\mathbf{K}}=\mathbf{K}^{-1} \mathbf{Q}_{T} \mathbf{K}^{+1} ; \quad \mathbf{K}^{ \pm 1}=\exp \left(\mp \mathrm{i} \text { thef } \sum_{i} \mathbf{I}_{i z}\right)
$$

Последнее преобразование не изменяет оператора начального состояния $\varrho_{T}(0)$. Некоторые же слагаемые гамильтонианов $\mathbf{H}_{d K}^{I I}$ и $\mathbf{H}_{K}^{I S}$ приобретают, однако, зависимость от времени с частотой $h_{e f}$. В дальнейшем предполагается, что частота $h_{e f}$ достаточно велика для того, чтобы не учитывать влияния зависящих от времени членов гамильтониана на состояние спин-системы. Это требует выполнения условия

$$
\left|h_{e f}\right| \gg\left|h_{L}\right| \text {. }
$$

В данном приближении

$$
\mathbf{H}_{d K}^{I I}=0,
$$

т. е. спин-локинг под магическим углом с достаточно интенсивным ВЧ полем эффективно исключает диполь-дипольное взаимодействие между I-спинами. Остаются, однако, диполь-дипольное и спин-спиновое взаимодействия между $S$ - и $I$-спинами, а также спин-спиновое взаимодействие между $I$-спинами.

В результате преобразований и принятых приближений из решения кинетического уравнения

$$
\mathrm{Q}(t)=\mathrm{e}^{-\mathrm{i} t \mathfrak{I}_{\left(\mathrm{H}_{\text {tock }}\right)} \mathrm{Q} T(0)}
$$

для состояния спин-суитемы в конце II этапа получаем выражение 


$$
\varrho_{K}(\tau)=(\operatorname{Sp} 1)^{-1}\left\{1-\beta \omega^{I} \sum_{i} \mathbf{I}_{i z}-\beta \omega^{S}\left(\mathbf{S}_{y} \cos \tau b-\mathbf{S}_{x} \sin \tau b\right)\right\},
$$

где

$$
b=\delta_{z z}^{\mathrm{S}}+\sqrt{\frac{1}{3}} \sum_{n}\left(J_{n}+B_{n}\right) \mathbf{I}_{n z} .
$$

При выводе (32) $\mathbf{H}_{\text {lock }}$ выбиралось в виде

$\mathbf{H}_{l o c k}=\sqrt{\frac{1}{3}} \sum_{i} \delta_{i z \mathbf{I}}^{I} \mathbf{I}_{i z}+\sum_{i<j} J_{i j} \mathbf{I}_{i z} \mathbf{I}_{j z}+\left[\delta_{z z}^{\mathrm{S}}+\sqrt{\frac{1}{3}} \sum_{n}\left(J_{n}+B_{n}\right) \mathbf{I}_{n z}\right] \mathbf{S}_{z}$,

т. е. в целях математического упрощения здесь, в дополнение к вышесказанному, опущены члены типа $J_{i j} \mathbf{I}_{i \pm} \mathbf{I}_{j \neq}$. Ясно, что это означает устранение одного из механизмов спиновой диффузии в системе $I$-спинов. Поскольку $J_{i j}-$ сравнительно небольшая величина (порядка $10 \mathrm{zц}$ в случае протонов в качестве $I$-спинов), то, вероятно, неточности, вызванные ошибками в параметрах ВЧ импульсов, превышают ошибки, связанные с неучетом спиновой диффузии за счет спин-спинового взаимодействия. Отметим, что в случае достаточно большого химического сдвига между $i$ и $j$ спинами пренебрежение членами $J_{i j} \mathbf{I}_{i \pm} \mathbf{I}_{j \mp}$ вполне оправдано.

\section{4. Об изменении состояния спин-системы во время регистрации}

Во время регистрации сигнала $S$-спина образец находится под влиянием ВЧ поля

$$
\overrightarrow{H(t)}=\vec{i} 2 H_{3} \cos \left[\omega^{I} t+\left(\omega^{I} \tau_{1}+\omega_{2} \tau\right)\right]
$$

За начало отсчета времени здесь принимается момент окончания спинлокинга,

Аналогично предыдущим этапам и в настоящем слущае пренебрегаем зависящими от времени членами в $\tilde{\mathbf{H}}_{z}^{I}$ и $\tilde{\mathbf{H}}_{z}^{\mathrm{S}}$ и учитываем прямое влияние ВЧ поля (35) только на $I$-спины

$$
\tilde{\mathbf{H}}_{r f}^{\mathrm{I}}=\sum_{i} h_{3 I} \mathbf{I}_{i x}, \quad \tilde{\mathbf{H}}_{r f}^{\mathrm{S}}=0 .
$$

Однако решение кинетического уравнения отличается от (18) не только начальным состоянием ( $\tilde{\varrho}(\tau)$ вместо $\left.\varrho_{0}\right)$, но и гамильтонианом, являющимся аргументом супероператора $\exp \left[-\mathrm{i} t \boldsymbol{\Omega}\left(\mathbf{H}_{\text {reg }}\right)\right]$. Так, интервал регистрации (теоретическая длительность от 0 до $\infty$ ) не отвечает условию типа (19), обязательному в случае пренебрежения влиянием. локальных полей. Поэтому, приступая к определению характера изменения состояния спин-системы, применим ко всем операторам во вращающихся координатах преобразавание

$$
\mathbf{Q}_{\mathbf{V}}=\mathbf{V}^{-1} \tilde{\mathbf{Q}} \mathbf{V}^{+1} ; \quad \mathbf{V}^{\neq 1}=\exp \left[\mp \mathrm{i} t\left(h_{3 I} \sum_{i} \mathbf{I}_{i x}+\delta_{z z}^{\mathrm{S}} \mathbf{S}_{z}\right)\right]
$$

и на основе предположения о том, что частота $h_{3 I}$ достаточно велика, пренебрежем всеми зависящими от времени членами в $\mathbf{H}_{V}$. В результате преобразования (37) начальное состояние не изменяется, а вид полу-

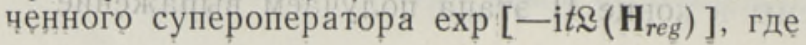




$$
\mathbf{H}_{\text {reg }}=\sum_{i<j}\left(J_{i j}-\frac{1}{3} B_{i j}\right) \overrightarrow{\mathbf{I}}_{i} \overrightarrow{\mathbf{I}}_{j},
$$

свидетельствует о развязке $S$ - от $I$-спинов. Соответственно, слагаемые $\tilde{\varrho}(\tau)$, содержащие только операторы $S$-спина, без изменения входят в состав QV $(t)$.

Далее заметим следующее:

1) В силу выражений

$$
\begin{gathered}
\cos \tau C_{n} \mathbf{I}_{n z}=\mathbf{1} \cos \frac{1}{2} \tau C_{n}, \quad \sin \tau C_{n} \mathbf{I}_{n z}=2 \mathbf{I}_{n z} \sin \frac{1}{2} \tau C_{n}, \\
C_{n}=\sqrt{\frac{1}{3}}\left(J_{n}+B_{n}\right)
\end{gathered}
$$

разложения $\cos \tau b$ и $\sin \tau b$ в (32) по параметрам ближайших соседей обладают только одним слагаемым (соответственно $1 \cos \tau \delta_{z z}^{\mathrm{s}} \boldsymbol{I}_{n} \cos \frac{1}{2} \tau C_{n}$ и $\left.1 \sin \tau \delta_{z z}^{\mathrm{s}} \boldsymbol{\Pi} \cos \frac{1}{2} \tau C_{n}\right)$, не содержащим по крайней мере одного оператора $\mathbf{I}_{n z}$. Операторы $\mathbf{I}_{n z}$ с произвольным значением $n$ содержатся в остальных слагаемых только в первой степени.

$\frac{2)}{\sqrt{\frac{1}{3}}}\left[\mathbf{I}_{n z}+\sqrt{2}\left(\mathbf{I}_{n y} \cos \tau \Omega+\mathbf{I}_{n z} \sin \tau \Omega\right)\right]$, при этом степень оператора с кон-
кретным индексом $n$ не изменяется.

3) На каждом шагу своего влияния супероператор $\exp \left[-i t \AA\left(\mathbf{H}_{\text {reg }}\right)\right]$ c (38) либо не изменяет комбинацию спиновых операторов в $е(\tau)$, либо заменяет по крайней мере одну компоненту $\mathbf{I}_{n k}$ на $\mathbf{I}_{n k}^{\prime}\left(k, k^{\prime}=x, y, z\right)$, не повышая ее степени.

Из вышесказанного можно сделать вывод, что «с точки зрения» сигнала

$$
S(t) \sim \gamma_{\mathrm{S}} \frac{d}{d t} \mathrm{Sp}\left\{\left[\mathbf{S}_{y} \cos \left[\omega_{0}^{S} t+\omega^{S}\left(\tau+\tau^{\prime}\right)\right]-\mathbf{S}_{x} \sin \left[\omega_{0}^{S} t+\omega^{S}\left(\tau+\tau^{\prime}\right)\right]\right] \varrho_{V}(t)\right\},
$$

индуцируемого $S$-спином в приемной катушке, направленной по $y$-оси лабораторных координат, существенной является только часть $\varrho_{V}^{S}(t)$ оператора плотности

$$
\varrho_{V}^{S}(t)=(\mathrm{Sp} \mathrm{1})^{-1} \beta \omega_{0}^{S}\left[\mathbf{S}_{y} \cos \tau \delta_{z z}^{\mathrm{S}}-\mathbf{S}_{x} \sin \tau \delta_{z z}^{S}\right] \boldsymbol{\Pi}_{n} \cos \frac{1}{2} \tau C_{n} .
$$

B (39)

$$
\omega_{0}^{S}=\omega^{S}-\delta_{z z}^{S}
$$

\section{5. О структуре спектра двукратного преобразования Фурье}

Наблюдаемый в данном эксперименте частотный спектр $S$-спина получается Фурье-преобразованием сигнала $S(t)$, накопленного во время регистрации. Однако при преобразовании (39) c @v $(t)$ в виде (40) появляются две трудности. Во-первых, значение интеграла на верхнем 
пределе интегрирования $(\infty)$ оказывается неопределенным, и, во-вторых, амплитуда появляющейся линии (центр в положении $\omega=\omega_{0}^{S}$ ) оказывается бесконечной. Причиной этих трудностей является игнорирование релаксации $S$-спина за время регистрации. Релаксация, естественно, приводит к нулевому значению интеграла на верхнем его пределе, что и будет принято в дальнейшем; амплитуда линии принимает также конечное значение. При учете релаксации (по крайней мере для случая, когда действие супероператора релаксации на $\mathrm{S}_{k}$ сводится к умножению на постоянную) амплитуда $A(\tau)$ линии была бы пропорциональна выражению

$$
\mathrm{i} \frac{\mathrm{Sp}\left(\mathbf{S}_{y}^{2}\right)}{\mathrm{Sp}(\mathbf{1})} \beta \omega^{S} \gamma_{s} \omega_{0}^{S} \mathrm{e}^{\mathrm{l}\left(\omega_{0}^{s} \tau+\omega^{s} \tau_{1}-\varphi\right)} \prod_{n} \cos \frac{1}{2} \tau C_{n}
$$

Из (42) видно, что величина амплитуды является функцией длительности $\tau$ интервала спин-локинга. Подвергая ее преобразованию Фурье, получаем частотный спектр двукратного преобразования $A(\omega)$.

При определении $A(\omega)$ исходя из (42) появляются аналогичные трудности, связанные с тем, что не учитывалась релаксация во время спин-локинта. Здесь значение интеграла на верхнем пределе также принимается равным нулю.

Фурье-преобразованием $A(\tau)$ выявляется положение центров линий в частотном спектре $A(\omega)$ :

$$
\omega=\omega_{0}^{S}+\frac{1}{2} \omega_{k}
$$

где величины $\omega_{k}$ - различные комбинации из всех возможных $C_{n}$. Например, в случае двух ближайших соседей $\omega_{1}=C_{1}+C_{2}, \omega_{2}=C_{1}-C_{2}$, $\omega_{3}=-C_{1}+C_{2}, \omega_{4}=-C_{1}-C_{2}$.

Следовательно, линии в спектре двукратного преобразования Фурье расположены симметрично относительно $\omega_{0}^{s}$ и число их $k=2^{n}$. Положения линий определяются константами связи между $S$-спином и его ближайшими соседями, т. е. рассмотренный эксперимент позволяет получать информацию об этих константах.

\section{Заключение}

Теоретически изучен частотный спектр ЯМР, полученный двукратным преобразованием Фурье сигнала редких ядер двухкомпонентной спиновой системы твердого образца. Рассмотренный экоперимент включает три этапа: импульсную подготовку состояния спиновой системы, спин-локинг многочисленных ядер под магическим углом и, наконеш, регистрацию сигнала редких ядер в условиях развязки их от многочисленных соседей.

Выяснено, что положения линий в спектре двукратного преобразования Фурье определяются константами связи между редкими ядрами и ближайшими к ним многочисленными ядрами. Таким образом, рассмотренный эксперимент позволяет изучать ближайшее окружение редких ядер в твердых телах.

\section{Л И Т Е Р А Т У Р А}

1. H a r tm a n n, S., H a h n, E. L.., Phys. Rev., 128, 2042 (1962).

2. Müller, L., Kumar, A., Baumann, T., Ernst, R. R., Phys. Rev. Letters, 32, 1402 (1974). 
3. Hester, R. K., Ackerman, J. L., Cross, V. R., Wa ugh, J. S., Phys. Rev. Letters, 34, 993 (1975).

4. Hester, R. K., A ckerman, J. L., N eff, B. L., W a ugh, J. S., Phys. Rev. Letters, 36, 1081 (1976).

5. W a u g h, J. S., частное сообщение.

6. L e e, M., G old burg, W. I., Phys. Rev. 140 A, 1261 (1965).

7. P ines , A., G i b by, M. G., W a u g h, J. S., J. Chem. Phys., 59, 569 (1973).

8. A u e, W. P., B a rt hold i, E., Ernst, R. R., J. Chem. Phys., 64, 2229 (1976).

9. H a e b e r l e n, U., W a u g h, J. S., Phys. Rev., 175, 453 (1968).

10. W a n g, C. H., Ram s h a w, J. D., Phys. Rev., B6, 3253 (1972).

Институт кибернетики

Академии наук Эстонской ССР
Поступила в редакцию $28 / \mathrm{X} 1976$

E. KUNDLA

\section{OHEST TMR-EKSPERIMENDIST, KUS ILMNEB MONOKRISTALLI MADALAKONTSENTRATSIOONILISTE TUUMADE INTERAKTSIOON NAABERTUUMADEGA}

Kirjeldatakse kahekomponendilise spinnsüsteemi (palju $I$ - ja vähe $S$-tuumi) käilumist katses, mis koosneb kolmest järjestikusest etapist, s. o. sobiva algseisu tekitamisest, $I$-tuumade nn. spin-locking'ust maagilise nurga all ja $S$-tuumade signaali registreerimisest nende samaaegse lahtisidestamisega I-tuumadest. Selgub, et joonte asendid registreeritud signaalist kahekordse Fourier' teisendusega saadud spektris sõltuvad $S$-tuuma ja tema naaber-I-tuumade vaheliste interaktsioonide konstantidest.

\section{E. KUNDLA}

\section{ON A SOLID STATE NMR EXPERIMENT IN WHICH A COUPLING APPEARS BETWEEN RARE AND NEIGHBOURING ABUNDANT NUCLEI}

The evolution of the state of a two-component (abundant $I$ and rare $S$ nuclei) spin system in a three-step experiment is described. The successive experimental steps are the initial state preparation, the magic angle spin-locking of $I$ nuclei, and the $S$-spin signal registration with simultaneous decoupling of $I$-nuclei. It becomes evident that the double Fourier spectra line positions depend on the coupling constants between $S$ and neighbouring $I$ nuclei. 